03

\title{
Построение промежуточных областей для обобщенного газа Ван-дер-Ваальса
}

\author{
(C) А.М. Блохин, ${ }^{1,2}$ А.Ю. Голдин ${ }^{2}$ \\ ${ }^{1,2}$ Институт математики им. С.Л. Соболева СО РАН, \\ 630090 Новосибирск, Россия \\ ${ }^{2}$ Новосибирский государственный университет, \\ 630090 Новосибирск, Россия \\ e-mail: blokhin@math.nsc.ru, goldinandrey@list.ru
}

(Поступило в Редакцию 3 ноября 2015 г.)

Обсужден подход к построению областей двуфазных состояний для реальных газов, состояние которых описано модифицированным уравнением Ван-дер-Ваальса в форме, предложенной Р.Л. Фогельсоном и E.P. Лихачевым.

\section{Введение}

В настоящей работе мы распространяем алгоритм построения областей двухфазных состояний или промежуточных областей по терминологии из [1] на уравнение состояния реального газа, предложенное в [1] (обобщенный газ Ван-дер-Ваальса).

Принято считать, что уравнение Ван-дер-Ваальса достаточно адекватно описывает жидкое и газообразное состояния реального вещества и переход из одного его состояния в другое [2-5]. При этом переход из одного состояния в другое на практике осуществляется через двуфазное состоянип [5]. Поскольку изотермы $T<T_{\mathrm{cr}}\left(T_{\mathrm{cr}}-\right.$ критическая температура) для газа Вандер-Ваальса сильно отличаются от экспериментальных изотерм реального газа, возникает проблема построения „истинных“ изотерм для газа Ван-дер-Ваальса (эти изотермы должны содержать кусок изобары, чтобы достаточно правильно описывать область перехода через двуфазное состояние вещества). Наш подход к построению истинных изотерм для газа Ван-дер-Ваальса и соответственно к построению областей двуфазных состояний изложен, например, в [6]. При этом мы активно используем принцип равных площадей Максвелла [3-5]. Чтобы проиллюстрировать разработанный нами алгоритм, мы используем конкретные численные результаты из [7,8], относящиеся к обтеканию клина обобщенным газом Вандер-Ваальса.

\section{1. Обтекание клина обобщенным газом Ван-дер-Ваальса. Предварительные сведения}

Как известно(см., например, [9-11]), уравнения газовой динамики имеют точное решение, которое соответствует стационарному обтеканию бесконечного клина равномерным потоком газа (рис. 1).

Структура этого решения такова. При $\theta>b(\theta=b-$ уравнение фронта ударной волны в полярной системе координат $r, \theta)$ :

$$
u=\hat{u}_{\infty}(\theta)=U_{\infty} \cos (\theta), \quad v=\hat{v}_{\infty}(\theta)=-U_{\infty} \sin (\theta),
$$

$U_{\infty}=$ const - скорость газа в набегающем потоке, $P=\hat{P}_{\infty}=f\left(\hat{\rho}_{\infty}, \hat{S}_{\infty}\right)=\mathrm{const}, \hat{\rho}=\rho_{\infty}=\mathrm{const}, \quad S=\hat{S}_{\infty}=$ $=$ const, $V=\hat{V}_{\infty}=\frac{1}{\hat{\rho}_{\infty}}=$ const - давление, плотность, энтропия и удельный объем в набегающем потоке газа, $c^{2}=\hat{c}_{\infty}^{2}=\frac{\partial f}{\partial \rho}\left(\hat{\rho}_{\infty}, \hat{S}_{\infty}\right)-$ квадрат скорости звука в набегающем потоке газа, причем $U_{\infty} \sin (b)>\hat{c}_{\infty}$, при $0<a<\theta<b<\frac{\pi}{2}$ ( $\theta=a-$ уравнение поверхностного клина):

$$
u=\hat{u}(\theta)=\hat{u}(a) \cos (s),
$$

$$
v=\hat{v}(\theta)=-\hat{u}(a) \sin (s), \quad s=\theta-a,
$$

$P=\hat{P}=f(\hat{\rho}, \hat{S})=\mathrm{const}\left(\hat{P}>\hat{P}_{\infty}\right), \rho=\hat{\rho}=\operatorname{const}\left(\hat{\rho}>\hat{\rho}_{\infty}\right)$, $S=\hat{S}=$ const $\left(\hat{S}>\hat{S}_{\infty}\right), V=\hat{V}=\frac{1}{\hat{\rho}}=\mathrm{const}-$ давление, плотность, энтропия и удельный объем в газе за ударной волной, $c^{2}=\hat{c}^{2}=\frac{\partial f}{\partial \rho}(\hat{\rho}, \hat{S})-$ квадрат скорости звука в газе за ударной волной, при $\theta=b$ выполняются условия

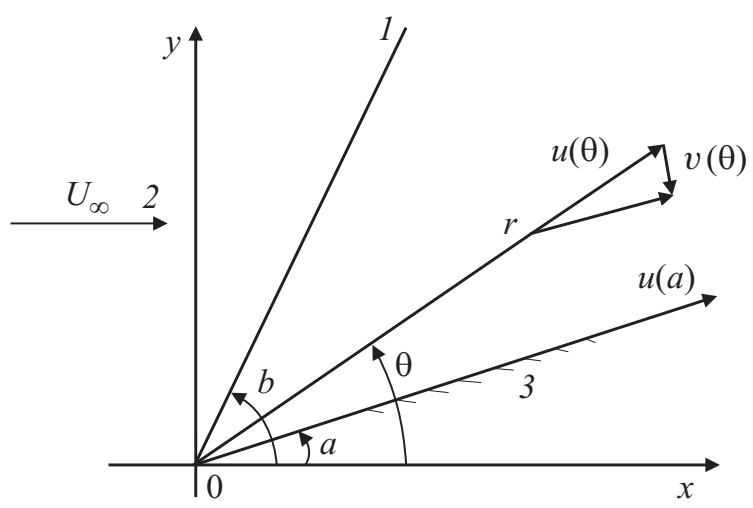

Рис. 1. Обтекание клина равномерным потоком вещества: 1 - ударная волна, $2-$ набегающий поток, 3 - клин. 
Рэнкина-Гюгонио на фронте ударной волны:

$$
\begin{aligned}
& \hat{u}(b)=\hat{u}_{\infty}(b), \\
& \left(\hat{v}(b)+U_{\infty} \sin (b)\right)^{2}+\left(\hat{P}-\hat{P}_{\infty}\right)\left(\hat{V}-\hat{V}_{\infty}\right)=0, \\
& E(\hat{V}, \hat{P})-E\left(\hat{V}_{\infty}, \hat{P}_{\infty}\right)+\left(\hat{V}-\hat{V}_{\infty}\right) \frac{\hat{P}+\hat{P}_{\infty}}{2}=0- \\
& \quad \text { адиабата Гюгонио, } \\
& \hat{\rho} \hat{v}(b)=\hat{\rho}_{\infty} \hat{v}_{\infty}(b)=-j(j>0-\text { поток газа } \\
& \quad \text { через разрыв }) .
\end{aligned}
$$

Здесь $u, v$ - компоненты вектора скорости газа в полярной системе координат, $P=f(\rho, S), e=E(V, P)-$ уравнения состояния газа, $e-$ внутренняя энергия газа.

Справедлив также первый закон термодинамики $[11,12]$ :

$$
T d S=d e+P d V
$$

где $T$ - температура газа. Если $P=P(V, T), e=e(V, T)$, $S=S(V, T)$, то из условия интегрируемости (2) мы придем к ограничению

$$
\frac{\partial e}{\partial V}=T^{2} \frac{\partial}{\partial T}\left(\frac{P}{T}\right) .
$$

Кроме этого,

$$
\frac{\partial S}{\partial T}=\frac{c_{V}}{T}, \quad \frac{d S}{d V}=\frac{1}{T}\left(\frac{\partial e}{\partial V}+P\right) .
$$

Здесь $c_{V}=\frac{\partial e}{\partial T}-$ удельная теплоемкость газа при постоянном объеме.

В настоящей работе мы будем иметь дело с обобщенным уравнением состояния газа Ван-дер-Ваальса $[1,7,8]$ :

$$
P=\frac{R T}{V-V_{0}}-\frac{A}{(V+C)^{k} T^{m}}, \quad V>V_{0}
$$

где $V_{0}, A, k, m>0, C$ - некоторые постоянные, $R-$ газовая постоянная. Заметим, что при $k=2, C=m=0$ уравнение (5) переходит в известное уравнение состояния газа Ван-дер-Ваальса, а при $C=m=0-$ в уравнение из работы [13].

Для уравнения состояния (5) мы последовательно можем получить [7, 8]:

$$
\begin{gathered}
e(V, T)=F(T)-\frac{m+1}{k-1} \frac{A}{(V+C)^{k-1} T^{m}}, \\
c^{2}=\frac{V^{2}\left(m P-(m+1) R T /\left(V-V_{0}\right)\right)^{2}}{T\left(F^{\prime}(T)+\frac{m(m+1)}{k-1}(V+C)\left(\frac{R}{V-V_{0}}-\frac{P}{T}\right)\right)} \\
+\frac{R T V^{2}}{\left(V-V_{0}\right)^{2}}-\frac{k V^{2}}{V+C}\left(\frac{R T}{V-V_{0}}-P\right) .
\end{gathered}
$$

Здесь $F(T)$ - некоторая функция.
Адиабату Гюгонио (1) для обобщенного газа Ван-дерВаальса сначала можно записать в таком виде:

$$
\begin{aligned}
& F(T)-\frac{m+1}{k-1}(V+C)\left(\frac{R T}{V-V_{0}}-P\right)-F\left(\hat{T}_{\infty}\right) \\
& +\frac{m+1}{k-1}\left(\hat{V}_{\infty}+C\right)\left(\frac{R \hat{T}_{\infty}}{\hat{V}_{\infty}-V_{0}}-\hat{P}_{\infty}\right)+\left(V-\hat{V}_{\infty}\right) \frac{P+\hat{P}_{\infty}}{2}=0,
\end{aligned}
$$

T. e.

$$
V=W\left(P ; \hat{P}_{\infty}, \hat{V}_{\infty}, V_{0}, C, A, m, k\right),
$$

где $T=T(P, V)-$ функция, которая находится из (5), $\hat{T}_{\infty}=T\left(\hat{P}_{\infty}, \hat{V}_{\infty}\right)$.

Путем громоздких выкладок из (8) получаем

$$
\begin{aligned}
& \frac{d W}{d P}\left(\hat{P} ; \hat{P}_{\infty}, \hat{V}_{\infty}, V_{0}, C, A, m, k\right) \\
& =-\left(\frac{\hat{V}-\hat{V}_{\infty}}{2}+\frac{F^{\prime}(\hat{T})\left(\hat{V}-V_{0}\right)}{\hat{r}}+\frac{m+1}{k-1}(\hat{V}+C)\right. \\
& \left.\times\left(1-\frac{R}{T}\right)\right) /\left(\left(\frac{\hat{P}+\hat{P}_{\infty}}{2}+\left(F^{\prime}(\hat{T})-\frac{m+1}{k-1}\right.\right.\right. \\
& \left.\times \frac{R(\hat{V}+C)}{\hat{V}-V_{0}}\right) \frac{\frac{R \hat{T}}{\hat{V}-V_{0}}+\frac{k}{V+C}\left(\hat{P}\left(\hat{V}-V_{0}\right)-R \hat{T}\right)}{\hat{r}} \\
& \left.-\frac{m+1}{k-1}\left(\frac{R \hat{T}}{\hat{V}-V_{0}}-\hat{P}\right)+\frac{m+1}{k-1}(\hat{V}+C) \frac{R \hat{T}}{\left(\hat{V}-V_{0}\right)^{2}}\right) \text {. }
\end{aligned}
$$

Здесь $\hat{r}=R+m\left(R-\frac{\hat{P}\left(\hat{V}-V_{0}\right)}{\hat{T}}\right), \hat{T}=T(\hat{P}, \hat{V})$.

В терминах агрегата

$$
\Delta=j^{2} \frac{d W}{d P}\left(\hat{P} ; \hat{P}_{\infty}, \hat{V}_{\infty}, V_{0}, C, A, m, k\right)
$$

ударная волна для обобщенного газа Ван-дер-Ваальса абсолютно (равномерно) устойчива, если [7,8]

$$
\Delta+1>0, \quad N<0,
$$

ударная волна нейтрально устойчива, если

$$
N, \mathscr{F}>0,
$$

где

$$
\begin{gathered}
N=\Delta-\frac{1-M^{2} \sin ^{2}(\delta)(1+\bar{\rho})}{1+M^{2} \sin ^{2}(\delta)(\bar{\rho}-1)}, \quad \mathscr{F}=1+2 M \sin (\delta)-\Delta, \\
M^{2}=\left(\frac{\hat{u}(a)}{\hat{c}}\right)^{2}, \quad \bar{\rho}=\frac{\hat{V}_{\infty}}{V}>1, \quad \delta=b-a,
\end{gathered}
$$

$M$ - число Маха в потоке вещества за ударной волной, $\hat{c}^{2}$ вычисляется по формуле (7). 
Замечание 1.1. Первое, второе и четвертое соотношения (1) можно заменить на следующие:

$$
\left.\begin{array}{l}
\hat{u}(a)=U_{\infty} \frac{\cos (b)}{\cos (\delta)}, \\
\hat{P}=\hat{P}_{\infty}+j U_{\infty} \frac{\sin (a)}{\cos (\delta)}, \\
\hat{\rho}=\operatorname{ctg}(\delta) \operatorname{tg}(b) .
\end{array}\right\}
$$

Удобно ввести следующие безразмерные параметры для уравнения состояния $(5)[7,8]$

$$
\begin{gathered}
\alpha=\frac{A R^{m}}{\hat{P}_{\infty}^{m+1} \hat{V}_{\infty}^{m+k}}, \quad \beta=\frac{V_{0}}{\hat{V}_{\infty}} \quad(0<\beta<1), \\
\tilde{\delta}=\frac{C}{\hat{V}_{\infty}}, \quad \gamma_{\infty}=1+\frac{R}{F^{\prime}\left(\hat{T}_{\infty}\right)}>1 .
\end{gathered}
$$

Тогда $[7,8]$

$$
\begin{aligned}
& \frac{\hat{P}}{\hat{P}_{\infty}}=1+\tilde{M}_{\infty}^{2} \frac{x y}{1+x y}, \quad \tilde{M}_{\infty}^{2}=M_{\infty}^{2} \frac{\hat{c}_{\infty}^{2}}{\hat{P}_{\infty} \hat{V}_{\infty}}, \\
& \frac{\hat{c}_{\infty}^{2}}{\hat{P}_{\infty} \hat{V}_{\infty}}=\frac{\Lambda_{\infty}}{(1-\beta)^{2}}+\frac{k}{1+\tilde{\delta}}\left(1-\frac{\Lambda_{\infty}}{1-\beta}\right) \\
& +\frac{\left(m-(m+1) \Lambda_{\infty} /(1-\beta)\right)^{2}}{\Lambda_{\infty}\left(\frac{1}{\gamma_{\infty}-1}+\frac{m(m+1)(1+\tilde{\delta})}{(k-1) \Lambda_{\infty}}\left(\frac{\Lambda_{\infty}}{(1-\beta)}-1\right)\right)}, \\
& \Lambda_{\infty}=(1-\beta)\left(1+\frac{\alpha}{(1+\tilde{\delta})^{k} \Lambda_{\infty}^{m}}\right) \text {, } \\
& \Lambda=(1-\beta \bar{\rho}) \frac{\hat{P} / \hat{P}_{\infty}+\alpha \bar{\rho}^{k} /(1+\tilde{\delta} \bar{\rho})^{k} \Lambda^{m}}{\bar{\rho}} \\
& \frac{c_{\infty}^{2}}{\hat{P}_{\infty} \hat{V}_{\infty}}=\frac{\Lambda_{\infty}}{(1-\beta \bar{\rho})^{2}}+\frac{k}{\bar{\rho}(1+\tilde{\delta} \bar{\rho})}\left(\frac{\hat{P}}{\hat{P}_{\infty}}-\frac{\Lambda \bar{\rho}}{1-\beta \bar{\rho}}\right) \\
& +\frac{\left(m \hat{P} / \hat{P}_{\infty}-(m+1) \Lambda \bar{\rho} /(1-\beta \bar{\rho})\right)^{2}}{\Lambda \bar{\rho}^{2}\left(\frac{F^{\prime}(\hat{T})}{R}+\frac{m(m+1)(1+\tilde{\delta} \bar{\rho})}{k-1}\left(\frac{1}{1-\beta \bar{\rho}}-\frac{\hat{P}}{P_{\infty}} \Lambda \bar{\rho}\right)\right)}, \\
& M^{2}=\tilde{M}_{\infty}^{2} \frac{1+\operatorname{tg}^{2}(\delta)}{1+x^{2}} \frac{\hat{P}_{\infty} \hat{V}_{\infty}}{\hat{c}^{2}} .
\end{aligned}
$$

Здесь $M_{\infty}^{2}=\frac{U^{2} \infty}{\hat{c}^{2} \infty}-$ квадрат числа Маха в набегающем потоке,

$$
\begin{gathered}
y=\operatorname{tg}(a), \quad x=\operatorname{tg}(b), \quad \hat{T}_{\infty}=\frac{\hat{P}_{\infty} \hat{V}_{\infty}}{R} \Lambda_{\infty}, \\
\hat{T}=\frac{\hat{P}_{\infty} \hat{V}_{\infty}}{R} \Lambda, \quad \bar{\rho}=\frac{x(1+x y)}{x-y} .
\end{gathered}
$$

Из формул, приведенных выше, следует, что агрегаты $\Lambda_{\infty}, \Lambda$ находятся неявным образом. Этот факт существенно усложняет численную реализацию соответствующего алгоритма.
Адиабата Гюгонио из (1), также (8) может быть переписана так:

$$
\begin{gathered}
\frac{F(\hat{T})-F\left(\hat{T}_{\infty}\right)}{\hat{P}_{\infty} \hat{V}_{\infty}}-\frac{y\left(1+x^{2}\right)}{x(1+x y)}\left(1+\tilde{M}_{\infty}^{2} \frac{x y}{2(1+x y)}\right) \\
+\frac{m+1}{k-1}\left((1+\tilde{\delta})\left(\frac{\Lambda_{\infty}}{1-\beta}-1\right)\right. \\
\left.-(1+\tilde{\delta} \bar{\rho})\left(\frac{\Lambda}{1-\beta \bar{\rho}}-\frac{\tilde{P}}{\tilde{P}_{\infty} \bar{\rho}}\right)\right)=0
\end{gathered}
$$

Далее мы конкретизируем вид функции $F(T)$. В отличие от [13] мы возьмем $F(T)$ в таком виде

$$
F(T)=c_{V}^{*} T\left(1+\frac{A_{1}}{2} T\right),
$$

где $c_{V}^{*}, A_{1}>0-$ некоторые постоянные (в работе [13] полагается, что $\left.A_{1}=0\right)$. Тогда $[7,8]$

$$
\begin{gathered}
c_{V}(T)=F^{\prime}(T)=c_{V}^{*}\left(1+A_{1} T\right), \\
\frac{c_{V}(\hat{T})}{R}=\frac{1}{\gamma^{*}-1}\left(1+\alpha_{T} \Lambda\right), \\
\gamma^{*}=1+\frac{R}{c_{V}^{*}}>1, \quad \alpha_{1}=A_{1} \frac{\hat{P}_{\infty} \hat{V}_{\infty}}{R}, \\
\frac{c_{V}\left(\hat{T}_{\infty}\right)}{R}=\frac{1}{\gamma_{\infty}-1}=\frac{1}{\gamma^{*}-1}\left(1+\alpha_{1} \Lambda_{\infty}\right),
\end{gathered}
$$

T.e.

$$
\begin{gathered}
\gamma^{*}>\gamma_{\infty} \quad \text { и } \quad \alpha_{1}=\frac{\gamma^{*}-\gamma_{\infty}}{\left(\gamma_{\infty}-1\right) \Lambda_{\infty}}, \\
\frac{F\left(\frac{\hat{P}_{\infty} \hat{V}_{\infty}}{R} \Lambda_{\infty}\right)}{\hat{P}_{\infty} \hat{V}_{\infty}}=\frac{\Lambda_{\infty}}{\gamma^{*}-1}\left(1+\frac{\alpha_{1}}{2} \Lambda_{\infty}\right), \\
\frac{F\left(\frac{\hat{P}_{\infty} \hat{V}_{\infty}}{R} \Lambda\right)}{\hat{P}_{\infty} \hat{V}_{\infty}}=\frac{\Lambda}{\gamma^{*}-1}\left(1+\frac{\alpha_{1}}{2} \Lambda\right)
\end{gathered}
$$

и соответственно соотношение (13) перепишется так

$$
\begin{aligned}
& Q(x)=\Lambda\left(1+\frac{\alpha_{1}}{2} \Lambda\right)-\Lambda_{\infty}\left(1+\frac{\alpha_{1}}{2} \Lambda_{\infty}\right)+\left(\gamma^{*}-1\right) \\
& \times\left[-\frac{y\left(1+x^{2}\right)}{x(1+x y)}\left(1+\tilde{M}_{\infty}^{2} \frac{x y}{2(1+x y)}\right)+\frac{m+1}{k-1}((1+\tilde{\delta})\right. \\
& \left.\left.\times\left(\frac{\Lambda_{\infty}}{1-\beta}-1\right)-(1+\tilde{\delta} \bar{\rho})\left(\frac{\Lambda}{1-\beta \bar{\rho}}-\frac{\hat{P}}{\hat{P}_{\infty} \bar{\rho}}\right)\right)\right]=0 .
\end{aligned}
$$

Следовательно, при заданных $a, \gamma_{\infty}, \gamma^{*}, M_{\infty}, \alpha, \beta, \tilde{\delta}$ находим корни уравнения $Q(x)=0$ и, значит, углы $b$. Замечание 1.2. Из (4) с учетом (14) получаем

$$
\begin{aligned}
\frac{S-S_{0}}{R}= & \ln \left(V-V_{0}\right)+\frac{1}{\gamma^{*}-1}\left(\ln T+A_{1} T\right) \\
& -\frac{m A}{R(k-1)(V+C)^{k-1} T^{m+1}},
\end{aligned}
$$

где $S_{0}-$ некоторая постоянная. 
Таблица 1.

\begin{tabular}{c|c|c|c|c|c|c}
\hline$M_{\infty}$ & \multicolumn{3}{|c|}{$\hat{T}_{\infty}<T_{\text {cr }}$} & $M_{\infty}$ & \multicolumn{3}{|c}{$\hat{T}_{\infty}<T_{\text {cr }}$} \\
\hline \multirow{2}{*}{3.0} & $b$ & $29.7077^{\circ}$ & $75.4234^{\circ}$ & 5.0 & $20.8432^{\circ}$ & $76.4641^{\circ}$ \\
& $M$ & 1.5665 & 0.5397 & & 1.9381 & 0.4967 \\
& $\Delta+1$ & 0.4568 & 0.8675 & & 0.6221 & 0.9476 \\
& $N$ & -0.6759 & -0.6762 & & -0.7164 & -0.6070 \\
& $\bar{\rho}^{-1}$ & 0.9599 & 0.9328 & & 0.9478 & 0.9285 \\
& $\hat{P} / \hat{P}_{\infty}$ & 3.1969 & 1.5013 & & 5.0854 & 4.2836 \\
& $Q$ & 0.2322 & 1.2382 & & 0.3971 & 2.4666 \\
& & $\left(\hat{T}<T_{\text {cr }}\right)$ & $\left(\hat{T}>T_{\text {cr }}\right)$ & & $\left(\hat{T}>T_{\text {cr }}\right)$ & $\left(\hat{T}>T_{\text {cr }}\right)$
\end{tabular}

Таблица 2.

\begin{tabular}{c|c|c|c|c|c|c}
\hline$M_{\infty}$ & \multicolumn{3}{|c|}{$\hat{T}_{\infty}<T_{\text {cr }}$} & $M_{\infty}$ & \multicolumn{2}{|c}{$\hat{T}_{\infty}<T_{\text {cr }}$} \\
\hline \multirow{3}{*}{3.0} & $b$ & $20.1368^{\circ}$ & $89.3231^{\circ}$ & 5.0 & $12.2378^{\circ}$ & $89.5217^{\circ}$ \\
& $M$ & 2.9039 & 0.5673 & & 4.7677 & 0.5212 \\
& $N$ & 0.0106 & 0.1117 & & 0.0192 & 0.2262 \\
& $\mathscr{F}$ & 3.8015 & 2.2763 & & 3.7105 & 2.1395 \\
& $\bar{\rho}^{-1}$ & 0.9463 & 0.4036 & & 0.9161 & 0.3235 \\
& $\hat{P} / \hat{P}_{\infty}$ & 1.1257 & 12.7892 & & 1.2071 & 38.1474 \\
& $Q$ & 0.0004 & 0.6433 & & -0.0006 & 1.5258 \\
& & $\left(\hat{T}<T_{\text {cr }}\right)$ & $\left(\hat{T}>T_{\text {cr }}\right)$ & & $\left(\hat{T}<T_{\text {cr }}\right)$ & $\left(\hat{T}>T_{\text {cr }}\right)$
\end{tabular}

Следуя [3-5] (см. также [1]), введем так называемые критические параметры для обобщенного газа Ван-дерВаальса:

$$
\begin{gathered}
A=\frac{(k+1)^{2}}{4 k}(1+v)^{k-1} R T_{\mathrm{cr}}^{m+1} V_{\mathrm{cr}}^{k-1}, \\
V_{0}=\frac{k-1-2 v}{k+1} V_{\mathrm{cr}}, \\
C=v V_{\mathrm{cr}}, \quad P_{\mathrm{cr}} V_{\mathrm{cr}}=\frac{k^{2}-1}{4 k(1+v)} R T_{\mathrm{cr}} .
\end{gathered}
$$

Отсюда получаем

$$
\begin{aligned}
& T_{\mathrm{cr}}=\frac{\hat{P}_{\infty} \hat{V}_{\infty}}{R} \tilde{l}, \tilde{l}=\left(\frac{\alpha l}{(\beta+\tilde{\delta})^{k-1}}\right)^{\frac{1}{m+1}} \\
& l=\frac{4 k(k-1)^{k-1}}{(k+1)^{k+1}}, \tilde{\delta}=\frac{\beta v(k+1)}{k-1-2 v}, \\
& V_{\mathrm{cr}}=\frac{\beta(k+1)}{k-1-2 v}, P_{\mathrm{cr}} V_{\mathrm{cr}}=\frac{k^{2}-1}{4 k(1+v)} \tilde{l}
\end{aligned}
$$

(параметры $P_{\text {cr }}, V_{\text {cr }}$ отнесены к $\hat{V}_{\infty}, \hat{P}_{\infty}$ ).

Замечание 1.3. Величины $k, m, v$ для различных реальных газов приведены в [1]. Кроме этого, напомним, что параметр $T_{\mathrm{cr}}$ характеризует собой ту изотерму, выше которой реальный газ нельзя превратить в жидкость. Заметим также, что неравенства

$$
\hat{T}_{\infty}, \hat{T} \lessgtr T_{\mathrm{cr}}
$$

сводятся к следующим:

$$
\Lambda_{\infty}, \Lambda \lessgtr \tilde{l}
$$

Наконец, из (15) получаем выражение для скачка энтропии на ударной волне

$$
\begin{aligned}
& \frac{\hat{S}-\hat{S}_{\infty}}{R}=Q(a, b) \\
& =\ln \left(\frac{1-\beta \bar{\rho}}{\bar{\rho}(1-\beta)}\right)+\frac{\ln \left(\frac{\Lambda}{\Lambda_{\infty}}\right)+\alpha_{1}\left(\Lambda-\Lambda_{\infty}\right)}{\gamma^{*}-1} \\
& \quad-\frac{m \alpha}{(k-1)}\left(\frac{\bar{\rho}^{k-1}}{(1+\bar{\rho} \delta)^{k-1} \Lambda^{m+1}}-\frac{1}{(1+\tilde{\delta})^{k-1} \Lambda_{\infty}^{m+1}}\right)
\end{aligned}
$$

Мы не будем приводить здесь многочисленные результаты расчетов для различных газов (они подробно обсуждаются в работах $[7,8])$. Отметим только, что далее нас будут интересовать ситуации, когда для вещества в набегающем потоке и после ударной волны выполнены неравенства $\hat{T}_{\infty}, \hat{T}<T_{\text {cr. }}$ Это означает, что вещество в таком случае может находиться в двухфазном состоянии [5]. Основная цель настоящей работы заключается в том, чтобы научиться строить области двуфазных состояний для обобщенного газа Ван-дер-Ваальса.

Замечание 1.4. Для иллюстрации приведены три таблицы. В табл. 1 для азота $(v=0, k=1.91, m=0.36,[1])$ приведены величины $b, M, \Delta+1, N, \bar{\rho}^{-1}, \hat{P} / \hat{P}_{\infty}, Q$ $(=Q(a, b),(17))$ при $a=1^{\circ}, \beta=0.9, \alpha=0.5, \gamma_{\infty}=2.1$, $\gamma^{*}=2.2$. 
Таблица 3.

\begin{tabular}{c|c|c|c|c|c|c}
\hline$M_{\infty}$ & \multicolumn{3}{|c|}{$\hat{T}_{\infty}<T_{\text {cr }}$} & $M_{\infty}$ & \multicolumn{2}{|c}{$\hat{T}_{\infty}<T_{\text {cr }}$} \\
\hline \multirow{2}{*}{3.0} & $b$ & $20.2065^{\circ}$ & $89.3387^{\circ}$ & 5.0 & $12.2806^{\circ}$ & $89.5460^{\circ}$ \\
& $M$ & 2.8875 & 0.5666 & & 4.7418 & 0.5159 \\
& $N$ & 0.0311 & 0.1222 & & 0.0421 & 0.8995 \\
& $\mathscr{F}$ & 3.8037 & 2.2738 & & 3.7134 & 2.1319 \\
& $\bar{\rho}^{-1}$ & 0.9465 & 0.3980 & & 0.9163 & 0.3122 \\
& $\hat{P} / \hat{P}_{\infty}$ & 1.1241 & 12.7013 & & 1.2044 & 38.1377 \\
& $Q$ & -0.0014 & 0.6435 & & -0.0021 & 1.5533 \\
& & $\left(\hat{T}<T_{\text {cr }}\right)$ & $\left(\hat{T}>T_{\text {cr }}\right)$ & & $\left(\hat{T}<T_{\text {cr }}\right)$ & $\left(\hat{T}>T_{\text {cr }}\right)$
\end{tabular}

Аналогично, в табл. 2 для фреона-12 ( $v=0.322$, $k=2.03, m=0.94[1])$ приведены величины $b, M, N, \mathscr{F}$, $\bar{\rho}^{-1}, \hat{P} / \hat{P}_{\infty}, Q$ при $a=1^{\circ}, \beta=0.01, \alpha=0.5, \gamma_{\infty}=2.1$, $\gamma^{*}=2.2$.

Наконец в табл. 3 для окиси углерода $(v=-0.091$, $k=1.8, m=0.28[1])$ приведены величины $b, M, N, \mathscr{F}$, $\bar{\rho}^{-1}, \hat{P} / \hat{P}_{\infty}, Q$ при $a=1^{\circ}, \beta=0.01, \alpha=0.5, \gamma_{\infty}=2.1$, $\gamma^{*}=2.2$.

Прокомментируем табл. 1-3. Как и для нормального газа, мы имеем два режима обтекания клина: с сильной ударной волной $(M<1)$ и со слабой ударной волной $(M>1)$. В табл. 1 для всех режимов ударная волна абсолютно устойчива. Для табл. 2 и 3 мы имеем интересную ситуацию: все режимы таковы, что ударные волны для них нейтрально устойчивы. Кроме этого, режим со слабой ударной волной является практически изэнтропическим. Судя по табл. 1-3, для режимов, соответствующих слабым ударным волнам, возможна ситуация, когда вещество как в набегающем потоке, так и после ударной волны может находиться не только в жидком или газообразном состоянии, но и в двуфазном состоянии.

\section{2. Построение областей двуфазных состояний}

Уравнение изотерм в наших обозначениях имеет следующий вид (см. уравнение состояния (5)):

$$
P=\frac{\lambda}{V-\beta}-\frac{\alpha}{\lambda^{m}(V+\tilde{\delta})^{k}},
$$

при этом (см. разд. 1$): \lambda \in\left(0, \lambda_{\mathrm{cr}}\right), \lambda_{\mathrm{cr}}=\tilde{l}$,

$$
\tilde{l}=\left[\frac{\alpha l}{(\beta+\tilde{\delta})^{k-1}}\right]^{\frac{1}{m+1}}, \quad l=\frac{4 k(k-1)^{k-1}}{(k+1)^{k+1}},
$$

$V_{\mathrm{cr}}=\frac{(k+1) \beta}{k-1-2 v}, \quad P_{\mathrm{cr}} V_{\mathrm{cr}}=\frac{\left(k^{2}-1\right)}{4 k(1+v)} \tilde{l}, \quad \tilde{\delta}=\frac{\beta v(k+1)}{k-1-2 v}$.

Напомним, что в (18) давление $P$ отнесено к $\hat{P}_{\infty}$, удельный объем $V$ к $\hat{V}_{\infty}$.

Типичная изотерма $A B C D$ изображена на рис. 2. Понятно, что она не являются истинной изотермой (на это указывает наличие $S$-образных участков). Истинна изотерма $T=\frac{\hat{P}_{\infty} \hat{V}_{\infty}}{R} \lambda, 0<\lambda<\tilde{l}\left(T<T_{\mathrm{cr}}\right)$ - это ломаная кривая $A B C D(B C-$ отрезок горизонтальной линии $P=P_{s}$ (изобары)). Область двуфазных состояний на рис. 2 для заданных величин $\alpha, \beta, v, m$ выделена штриховой кривой, проходящей через точки $M, B, K$, $C, N$.

Приступим теперь к определению параметров $P_{s}$, $V_{B}, V_{C}$ (рис. 2). Привлекая правило равных площадей Максвелла [3-5] и уравнение (18), получим следующую нелинейную алгебраическую систему для нахождения параметров $V_{B}, V_{C}, P_{s}\left(V_{B} \neq V_{C}\right)$ :

$$
\begin{aligned}
P_{s} \Delta= & \lambda \ln \left(\frac{V_{C}-\beta}{V_{B}-\beta}\right) \\
& -\frac{\alpha}{\lambda_{m}} \frac{\left(V_{C}+\tilde{\delta}\right)^{k-1}-\left(V_{B}+\tilde{\delta}\right)^{k-1}}{\left(V_{B} V_{C}+\tilde{\delta}\left(V_{B}+V_{C}\right)+\tilde{\delta}^{2}\right)^{k-1}(k-1)}, \\
P_{S}= & \frac{\lambda}{V_{B}-\beta} \\
& -\frac{\alpha}{\lambda_{m}} \frac{1}{\left(V_{B}+\tilde{\delta}\right)^{k}}=\frac{\lambda}{V_{C}-\beta}-\frac{\alpha}{\lambda^{m}} \frac{1}{\left(V_{C}+\tilde{\delta}\right)^{k}},
\end{aligned}
$$

где $\Delta=V_{C}-V_{B}$.

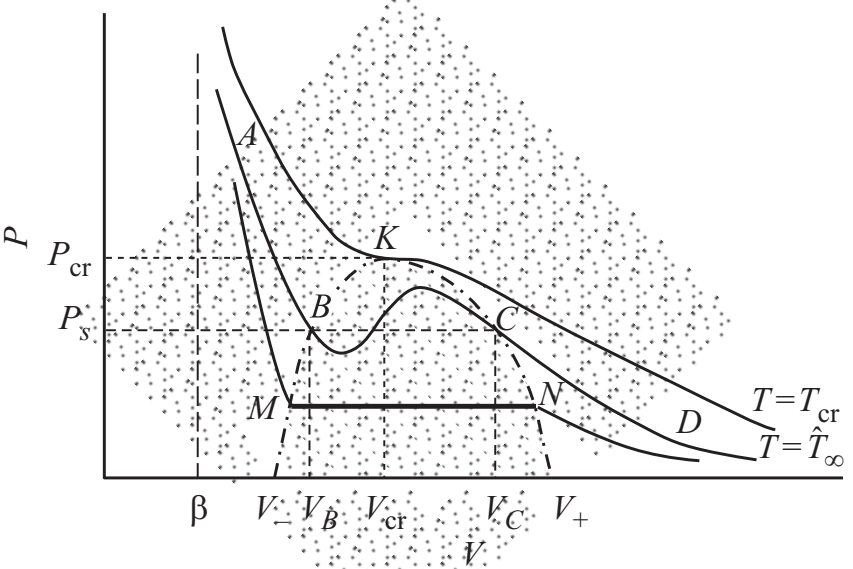

Рис. 2. Область двуфазных состояний. 
Применяя лемму Адамара [14], перепишем систему (19), (20) так

$$
\begin{gathered}
\frac{\lambda^{m+1}}{V_{B} V_{C}-\beta\left(V_{C}+V_{B}\right)+\beta^{2}}=\alpha k \frac{\int_{0}^{1}\left[s \Delta+V_{B}+\tilde{\delta}\right]^{k-1} d s}{\left(V_{B} V_{C}+\tilde{\delta}\left(V_{B}+V_{C}\right)+\tilde{\delta}^{2}\right)^{k}}, \\
\lambda^{m+1}\left\{\frac{1}{V_{B}-\beta}+\frac{1}{\Delta} \ln \left(\frac{V_{B}-\beta}{V_{C}-\beta}\right)\right\} \\
=\alpha \frac{\left(V_{C}+\tilde{\delta}\right)^{k-1}-\left(V_{B}+\tilde{\delta}\right) \int_{0}^{1}\left[s \Delta+V_{B}+\tilde{\delta}\right]^{k-2} d s}{\left(V_{B}+\tilde{\delta}\right)\left(V_{B} V_{C}+\tilde{\delta}\left(V_{B}+V_{C}\right)+\tilde{\delta}\right)^{k-1}} .
\end{gathered}
$$

Следуя [6], вместо $V_{B}, V_{C}$ введем следующие параметры:

$$
V_{C}=y \frac{1+\sqrt{1-q}}{2}, V_{B}=y \frac{1-\sqrt{1-q}}{2}, q=\frac{4 x}{y^{2}}(<1) \text {, }
$$

где величины $y, x>0$. Тогда из (21) получаем

$$
x=\beta y-\beta^{2}+\frac{\lambda^{m+1}(x+\tilde{\delta} y+\tilde{\delta} 2)^{k}}{\alpha k \int_{0}^{1}\left[s \Delta+V_{B}+\tilde{\delta}\right]^{k-1} d s},
$$

причем

$$
\begin{aligned}
\int_{0}^{1}[s & \left.\Delta+V_{B}+\tilde{\delta}\right]^{k-1} d s \\
& =\int_{0}^{1}\left[s \sqrt{y^{2}-4 x}+\frac{y-\sqrt{y^{2}-4 x}+2 \tilde{\delta}}{2}\right]^{k-1} d s .
\end{aligned}
$$

Замечание 2.1. Вышеупомянутая лемма Адамара [14] для нашего случая формулируется так:

$$
\left(V_{C}+\tilde{\delta}\right)^{\mu}-\left(V_{B}+\tilde{\delta}\right)^{\mu}+\mu \Delta \int_{0}^{1}\left[s \Delta+V_{B}+\tilde{\delta}\right]^{\mu-1} d s .
$$

Здесь $\mu$ - некоторая постоянная величина.

Находя зависимость $x=x(y)$ и подставляя ее в соотношение (22), получим

$$
\begin{aligned}
& \tilde{Q}(y)=Q(x(y), y) \\
& =\lambda^{m+1}\left\{\frac{1}{V_{B}-\beta}+\frac{1}{\sqrt{y^{2}-4 x}} \ln \left(\frac{V_{V}-\beta}{V_{C}-\beta}\right)\right\}-\alpha \\
& \times \frac{\left(V_{C}+\tilde{\delta}\right)^{k-1}-\left(V_{B}+\tilde{\delta}\right) \int_{0}^{1}\left[s \sqrt{y^{2}-4 x}+\frac{y-\sqrt{y^{2}-4 x}+2 \tilde{\delta}}{2}\right]^{k-2} d s}{\left(V_{B}+\tilde{\delta}\right)\left(x+y \tilde{\delta}+\tilde{\delta}^{2}\right)^{k-1}}=0 .
\end{aligned}
$$

Определяя нули функции $\tilde{Q}(y)$, находим затем параметры $V_{B}, V_{C}, P_{S}$.
Таким образом, наш алгоритм построения областей двуфазных состояний заключается в следующем: мы для уравнения состояния (18), $\lambda \in\left(0, \lambda_{\mathrm{cr}}\right)$ и конкретных значений параметров $\alpha, \beta, v, m$ строим сначала „истинные“ изотермы, а затем зависимости

$$
V=V_{B}(P), \quad 0<P<P_{\mathrm{cr}}
$$

и

$$
V=V_{C}(P), \quad 0<P<P_{\mathrm{cr}} .
$$

Эти кривые и ограничивают искомую область двуфазных состояний для данных $\alpha, \beta, v, m$. Для того чтобы определить, в каком состоянии находится вещество (либо в набегающем потоке, либо после ударной волны), мы должны определить положение точек $(1,1),\left(\frac{\hat{P}}{P_{\infty}}, \frac{1}{\bar{\rho}}\right)$ относительно построенной области. Для параметров $\alpha, \beta$,

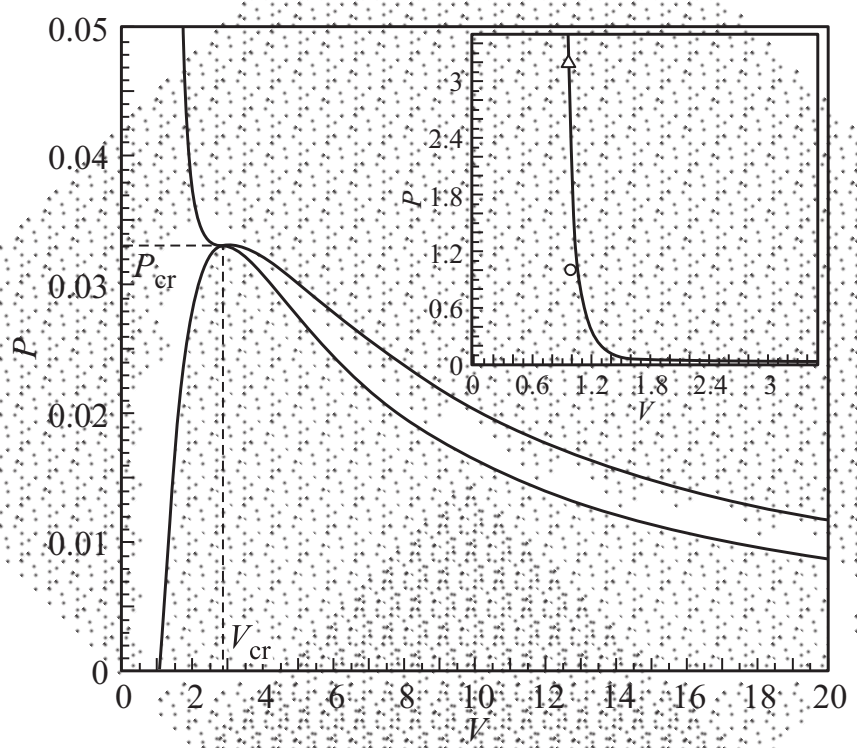

Рис. 3. Область двуфазных состояний для азота (табл. 1).

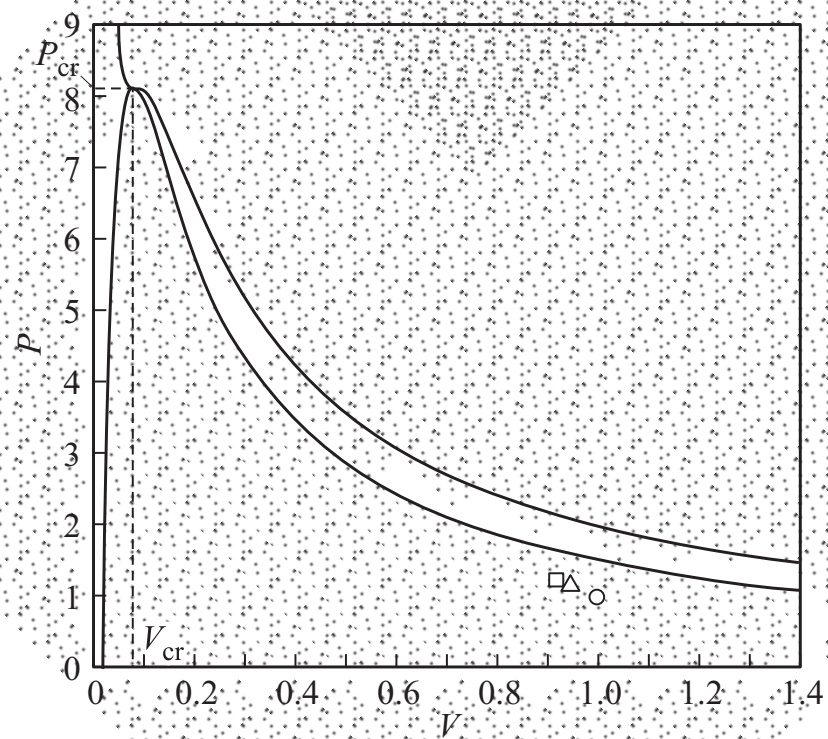

Рис. 4. Область двуфазных состояний для фреона-12 (табл. 2). 


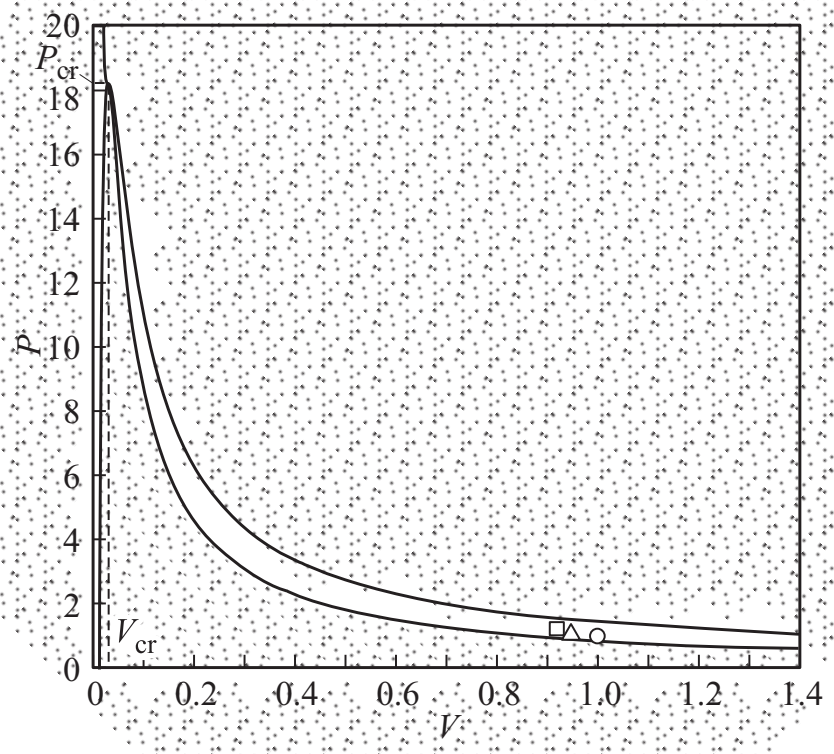

Рис. 5. Область двуфазных состояний для окиси углерода (табл. 3).

$v, m$, взятых из табл. 1-3, области двуфазных состояний изображены на рис. 3-5 (кружочком обозначена точка $(1,1)$, треугольником - точка $\left(\frac{\hat{P}}{P_{\infty}}, \frac{1}{\bar{\rho}}\right)$ при $M_{\infty}=3$, квадратиком - точка $\left(\frac{\hat{P}}{P_{\infty}}, \frac{1}{\bar{\rho}}\right)$ при $\left.M_{\infty}=5\right)$.

\section{Заключительные замечания}

Прокомментируем рис. 3-5. Для азота вещество в набегающем потоке (табл. $1, M_{\infty}=3$ ) находится в жидком состоянии (рис. 3). Вещество после ударной волны также находится в жидком состоянии (точка $\left(\frac{\hat{P}}{\hat{P}_{\infty}}, \bar{\rho}^{-1}\right)$ при $M_{\infty}=3$ обозначена треугольником). Для фреона-12 вещество как в набегающем потоке, так и после ударной волны находится в двуфазном состоянии (рис. 4), причем поскольку все точки расположены близко от правой границы области двуфазных состояний, в веществе больше насыщенного пара, чем жидкости. Для окиси углерода (рис. 5) вещество как в набегающем потоке, так и после ударной волны, скорее, находится в газообразном состоянии.

Авторы признательны А.С. Бычкову за проведенные численные расчеты, касающиеся обтекания клина обобщенным газом Ван-дер-Ваальса.

\section{Список литературы}

[1] Фогельсон Р.Л., Лихачев Е.Р. // ЖТФ. 2004. Т. 74. Вып. 7. C. $129-130$

[2] Вукалович М.П., Новиков Н.Н. Уравнение состояния реальных газов. М.; Л.: Госэнергоиздат, 1948. 340 с.

[3] Тер Хаар Д., Вергеланд Г. Элементарная термодинамика. М.: Мир, 1968. 220 с.

[4] Кубо Р. Термодинамика. М.: Мир, 1970. 304 с.
[5] Матвеев А.Н. Молекулярная физика. М.: Оникс, Мир и образование, 2006. $360 \mathrm{c}$.

[6] Blokhin A.M., Goldin A.Yu. Construction of domains of twophase states for a real substance. International Conference on the Methods of Aerophysical Research, June 27-July 3, 2016. Perm, Russia: Abstracts. Pt I/Ed. V.M. Fomin. P. 29-30.

[7] Блохин А.М., Ткачев Д.Л. // ЖТФ. 2015. Т. 85. Вып. 7. C. $20-30$.

[8] Блохин А.М., Бычков А.С., Мякишев В.О. О выполнении условия Лопатинского в задаче об обтекании клина нормальным газом и газом Ван-дер-Ваальса. Препринт / Институт математики СО РАН, № 280, Новосибирск, 2012. 58 с.

[9] Овсянников Л.В. Лекции по основам газовой динамики. М.: Наука, 1981. 368 с.

[10] Лойиянский Л.Г. Механика жидкости и газа. М.: Наука, 1978. $736 \mathrm{c}$.

[11] Седов Л.Н. Механика сплошной среды. Т. 1. М.: Наука, 1970. $492 \mathrm{c}$.

[12] Рождественский Б.Л., Яненко Н.Н. Системы квазилинейных уравений. М.: Наука, 1978. 688 с.

[13] Конюхов А.В., Лихачев А.Р., Фортов В.Е., Опарин А.М., Анисимов С.Н. Нейтрально устойчивые ударные волны в термодинамически неидеальных средах: теория и вычислительный эксперимент. Тр. Междунар. конф. „ІХ Забабахинские научные чтения“. 2007.

[14] Карташев А.П., Рождественский Б.Л. Обыкновенные дифферециальные уравнения и основы вариационного исчисления. М.: Наука, 1976. 256 с. 